\title{
Treatments for obstructive hydrocephalus secondary to malignant midline intracranial tumors during the perioperative period $-a$ retrospective study of 372 pediatric patients from a single institution
}

\section{Yunwei Ou}

Beijing Tiantan Hospital, Capital Medical University

Kaiyu Fan ( $\sim$ fankaiyu118@163.com )

Beijing Tiantan Hospital,Capital Medical University https://orcid.org/0000-0002-8854-3004

Zhiming Liu

Beijing Tiantan Hospital, Capital Medical University

Zhiyi Liao

Beijing Tiantan Hospital, Capital Medical University

Heng Zhang

Beijing Tiantan Hospital, Capital Medical University

\section{Zhicen Li}

Beijing Tiantan Hospital, Capital Medical University

Wenjian Zheng

Beijing Tiantan Hospital, Capital Medical University

\section{Xueyi Guan}

Beijing Tiantan Hospital, Capital Medical University

Jian Gong

Beijing Tiantan Hospital, Capital Medical University https://orcid.org/0000-0003-3224-9997

\section{Research Article}

Keywords: obstructive hydrocephalus, midline tumors, ventriculoperitoneal shunt, endoscopic third ventriculostomy

Posted Date: May 13th, 2021

DOl: https://doi.org/10.21203/rs.3.rs-476777/v1 
License: (c) (i) This work is licensed under a Creative Commons Attribution 4.0 International License. Read Full License 


\section{Abstract \\ Objective}

Common treatments for obstructive hydrocephalus caused by malignant midline intracranial tumors during the perioperative period include ventriculoperitoneal shunt (VPS) placement/endoscopic third ventriculostomy (ETV) and direct tumor resection, but which of these treatments is superior remains unclear. The purpose of this study is to explore the management of hydrocephalus during the perioperative period and subsequent outcomes.

\section{Methods}

Data from 372 patients with obstructive hydrocephalus due to malignant midline intracranial tumors under the age of 18 years referred to the Department of Pediatric Neurosurgery at Beijing Tiantan Hospital between January 2018 and September 2019 were collected. We also collected their clinical features and outcomes for further statistical analysis.

\section{Results}

A total of 372 pediatric patients were treated for obstructive hydrocephalus. In total, 215 patients underwent preoperative VPS placement; the effectiveness of preoperative VPS placement was $98.1 \%$ $(211 / 215)$, and the mean duration of relapse was $63.5 \pm 15.7$ days. Forty children underwent ETV before tumor removal; the effectiveness of preoperative ETV was $90.0 \%(36 / 40)$, and the mean duration of relapse was $53.8 \pm 44.9$ days. A total of 117 patients underwent direct tumor resection after being diagnosed; the recurrence rate of hydrocephalus was $20.5 \%$ (24/117), and the mean duration of relapse was $125.0 \pm 170.8$ days. There was a significant difference between preoperative VPS placement followed by resection and postoperative VPS placement and preoperative ETV followed by resection and postoperative VPS placement $(p=0.013)$.

\section{Conclusion}

Malignant midline intracranial tumors in pediatric patients usually lead to obstructive hydrocephalus, and preoperative intervention for hydrocephalus (VPS or ETV) will improve patient outcomes. The optimal management strategy for obstructive hydrocephalus due to malignant midline intracranial tumors is preoperative VPS placement or ETV due to their low hydrocephalic recurrence rates and high effectiveness.

\section{Introduction}


Hydrocephalus is the most common condition treated by pediatric neurosurgeons[10]. Obstructive hydrocephalus (also known as noncommunicating hydrocephalus) occurs when there is obstructed cerebrospinal fluid (CSF) flow within the ventricular system[15], especially in the intraventricular, paraventricular[16] and other midline regions. Therefore, obstructive hydrocephalus is one of the most common complications of midline tumors, leading to an abnormal accumulation of CSF in the central nervous system[20] and eventually increased intracranial pressure (ICP). For malignant midline tumors, subsequent chemotherapy and radiotherapy are often required after resection, introducing more considerations about how to relieve the recurrence of hydrocephalus.

To relieve the symptoms of obstructive hydrocephalus caused by malignant midline intracranial tumors, neurosurgeons often prefer surgery to remove the tumors and recover CSF circulation. Nevertheless, avoiding acute encephalocele and ensuring the recovery CSF circulation during surgery is not guaranteed. Furthermore, how to prevent hydrocephalus relapse during subsequent therapy is still unclear.

Thus, ventriculoperitoneal shunts (VPSs) have become one of the conventional treatments[7, 26] and have undergone many advances since their first use in the mid-1950s[24]. However, the potential complications were significant, including foreign material implantation, shunt malfunction, infections, and cognitive impairment, which remained a constant source of concern for the child, parents, and family[4, 18, 23, 26].

In the 1990s, endoscopic third ventriculostomy (ETV) emerged as an effective alternative treatment for hydrocephalus, along with improvements in endoscopy and camera technologies[4,9]. The primary advantage of ETV is freedom from foreign material implantation, but this approach cannot ensure whether the stoma closes early.

Therefore, which of these treatments is superioris still widely discussed and remains unclear. Neurosurgeons are usually expected to rely on their experience[8]. To address this problem, we reviewed 372 pediatric patients who were treated for obstructive hydrocephalus at a single center to describe the management of hydrocephalus during the perioperative period and the subsequent outcomes.

\section{Methods}

\section{Clinical criteria}

Over 1,000 pediatric patients are admitted to the Department of Pediatric Neurosurgery, Beijing Tiantan Hospital every year; more than $80 \%$ of them are children with intracranial tumors, and approximately half are complicated with obstructive hydrocephalus. In total, we retrospectively reviewed 372 consecutive pediatric patients during the study period from January 2018 to September 2019. All patients had undergone preoperative and postoperative neurological and radiological evaluations. The inclusion criteria were as follows: 1 ) under 18 years of age; 2 ) initial neuroimaging showed solid midline intracranial tumors (Ommaya reservoirs rather than ETV/VPS placement were preferred for cystic tumors), complicated with enlarged ventricles as well as paraventricular edema (Fig 1);3) postoperative 
pathology including glioma, germ cell tumors, medulloblastoma, anaplastic ependymoma, etc.; and 4) initial VPS placement or ETV was implemented preoperatively or the tumor was directly resected after diagnosis to relieve hydrocephalus. Clinical characteristics were collected from medical records, such as age at the first operation, sex, tumor location, pathology, preoperative and postoperative therapeutic schedule, date of the initial procedure, date of resection, date of every subsequent procedure, date of hydrocephalus relapse, date of the last follow-up, and outcomes. Regular follow-up data regarding postoperative symptoms and outcomes were obtained from 1 year to 3 years after surgery via telephone. The criterion to define an effective operation that relieved hydrocephalus was mainly the absence of signs or symptoms of increased ICP, including an improved level of consciousness, resolution of eye movement abnormalities, and relief from headache, vomiting, and urinary incontinence. Follow-up neuroimaging was not considered crucial because on imaging, the ventricles often do not show a remarkable reduction in size postoperatively compared to preoperatively[4]. In contrast, the criterion for hydrocephalus recurrence was relapse of the signs and symptoms above, and neuroimaging indicated absolute enlargement of the ventricles.

\section{Statistical analysis}

For clinical parameters, continuous variables are presented as means ( \pm standard deviations), and categorical variables are presented as numbers of patients and frequencies. The differences in clinical parameters were analyzed by t-tests or chi-square tests. SPSS software version 17.0.0 (IBM Corp., Armonk, New York, USA) was used for statistical analyses. A $p<0.05$ was used as a threshold for statistical significance, and all tests were two-tailed.

\section{Results}

\section{Clinical characteristics}

A total of 372 pediatric patients were treated for obstructive hydrocephalus at our department from January 2018 to September 2019, and their conditions were all caused by malignant midline intracranial tumors. There was an obvious preponderance of boys (65.1\%; Table 1$)$. The mean age at the first surgery was $7.6 \pm 4.1$ years. The midline tumors originated from three locations, including suprasellar $(9.7 \%)$, pineal region (26.1\%), and the fourth ventricle (64.2\%). Moreover, the most common pathology was medulloblastoma (36.0\%), followed by germ cell tumor (18.8\%), glioma (18.0\%), other types of tumors (17.5\%), and anaplastic ependymoma (9.7\%).

Furthermore, the sex ratio $(0.7,4.4,1.6$, respectively, $p<0.001$; Table 2$)$, as well as the mean age at first surgery $(7.0 \pm 4.3,9.4 \pm 4.1,7.0 \pm 3.8$, respectively, $p<0.001)$, was significantly different among the three locations of suprasellar, pineal region and the fourth ventricles. The main pathology in suprasellar was glioma (66.7\%), whereas the most common pathology in the pineal region was germ cell tumors (63.9\%), and the fourth ventricle showed a predominance for medulloblastoma (56.1\%), which led to significant differences in pathology among the three locations $(p<0.001)$. 
Among the 372 patients, 117 underwent direct excision after diagnosis (31.4\%; Table 3), 40 underwent ETV preoperatively (10.8\%), and 215 underwent VPS placement (57.8\%). After tumor resection, 3 children underwent ETV ( 1 tumor in suprasellar (5.6\%), 2 in the fourth ventricle $(0.8 \%)$ ), and 31 underwent VPS placement (6 tumors in the suprasellar are (16.7\%), 9 in the pineal region (9.3\%), 16 in the fourth ventricle $(6.7 \%))$ due to the recurrence of hydrocephalus.

\section{Outcomes of initial VPS placement or ETV}

VPS placement was performed as the first-line treatment in 215 patients with obstructive hydrocephalus. In our analysis, sex and age at the first surgery were significantly different among the three locations ( $p=$ 0.021 and 0.010 , respectively; Table 4). The outcomes of these 215 patients were optimistic; only 4 children experienced recurrence of hydrocephalus because of shunt blockage (2 tumors in the suprasellar and 1 in the fourth ventricle underwent VPS placement again after resection, and 1 from the fourth ventricle underwent ETV). The effectiveness of preoperative VPS placement was $98.1 \%$ (211/215), and the mean duration of relapse was $63.5 \pm 15.7$ days.

Forty children underwent ETV before tumor resection; sex and age at the first surgery were not significantly different among the locations ( $p=0.069$ and 0.078 , respectively; Table 5 ). Hydrocephalus recurred in 3 patients with germ cell tumors and 1 with anaplastic ependymoma, so VPS placement was performed after tumor resection ( 3 from the pineal region and 1 from the fourth ventricle), so the effectiveness of preoperative ETV was $90.0 \%$ (36/40), and the mean duration of relapse was $53.8 \pm 44.9$ days. Therefore, the effectiveness of preoperative VPS placement and ETV were both over $90 \%$, but there was a significant difference between preoperative VPS placement followed by resection and postoperative VPS placement and preoperative ETV followed by resection and postoperative VPS placement $(p=0.013)$.

\section{Outcomes of direct resection}

Furthermore, 117 patients underwent direct resection after diagnosis instead of VPS placement or ETV. The sex ratio was significantly different among the three locations $(p=0.003$; Table 6$)$, while age at the first surgery was not different $(p=0.155)$. According to our statistics, $20.5 \%(24 / 117)$ of patients (seven gliomas, seven medulloblastomas, four germ cell tumors, four anaplastic ependymomas, and two other malignant tumors) underwent VPS placement after tumor resection because of recurrence of hydrocephalus. Interestingly, two (1.7\%) of these patients underwent ETV after the hydrocephalus recurred, but the symptoms were not relieved, and they finally underwent VPS placement to relieve their condition. The mean duration of relapse was $125.0 \pm 170.8$ days, the maximum and minimum durations were 710 and 7 days, respectively, and the median was 42 days. It is worth emphasizing that 22 of the 24 patients suspended their treatment due to sudden hydrocephalus relapse during chemoradiotherapy.

\section{TABLE 1. Summary of the clinical characteristics of 372 pediatric patients with an initial presentation of hydrocephalus caused by malignant midline intracranial tumors}




\begin{tabular}{lc}
\hline Clinical characteristics & Patients n (\%) \\
\hline Gender & $242(65.1)$ \\
$\quad$ Male & $130(34.9)$ \\
Female & $7.6 \pm 4.1$ \\
Age (years) & \\
Locations of tumor & $36(9.7)$ \\
$\quad$ Suprasellar & $97(26.1)$ \\
Pineal region & $239(64.2)$ \\
Fourth ventricle & \\
Pathology & $67(18.0)$ \\
$\quad$ Glioma & $70(18.8)$ \\
Germ cell tumor & $134(36.0)$ \\
$\quad$ Medulloblastoma & $39(9.7)$ \\
Anaplastic ependymoma & $65(17.5)$ \\
$\quad$ Others &
\end{tabular}

TABLE 2. Clinical characteristics of 372 pediatric patients with hydrocephalus caused by malignant midline intracranial tumors in 3 different locations

\begin{tabular}{lcccc}
\hline \multirow{2}{*}{ Clinical characteristics } & \multicolumn{3}{c}{ Location of tumors (n) } & \multirow{2}{*}{$p$} \\
\cline { 2 - 4 } & $\begin{array}{c}\text { Suprasellar } \\
(36)\end{array}$ & $\begin{array}{c}\text { Pineal region } \\
(97)\end{array}$ & $\begin{array}{c}\text { Fourth ventricle } \\
(239)\end{array}$ & \\
\hline Gender & $15(41.7)$ & $79(81.4)$ & $148(61.9)$ & $<0.001$ \\
$\quad$ Male & $21(58.3)$ & $18(18.6)$ & $91(38.1)$ & \\
Female & $7.0 \pm 4.3$ & $9.4 \pm 4.1$ & $7.0 \pm 3.8$ & $<0.001$ \\
Age (years) & $24(66.7)$ & $6(6.2)$ & $37(15.5)$ & \\
Pathology & $7(19.4)$ & $62(63.9)$ & $1(0.4)$ & \\
$\quad$ Glioma & $0(0.0)$ & $0(0.0)$ & $134(56.1)$ & \\
Germ cell tumor & $0(0.0)$ & $2(2.1)$ & $34(14.2)$ & \\
Medulloblastoma & $5(13.9)$ & $27(27.8)$ & $33(13.8)$ & \\
Anaplastic & & &
\end{tabular}

TABLE 3. Treatment for 372 pediatric patients with hydrocephalus caused by tumors located in 3 different regions

\begin{tabular}{|c|c|c|c|}
\hline \multirow[b]{2}{*}{ Treatments } & \multicolumn{3}{|c|}{ Location of tumors (n) } \\
\hline & $\begin{array}{c}\text { Suprasellar } \\
(36)\end{array}$ & $\begin{array}{c}\text { Pineal region } \\
(97)\end{array}$ & $\begin{array}{c}\text { Fourth ventricle } \\
\text { (239) }\end{array}$ \\
\hline 1170 & $14(36.1)$ & $24(24.7)$ & $79(33.1)$ \\
\hline $\begin{array}{l}\text { Pre } \\
\text { Pre }\end{array}$ & $21(6$ & $\begin{array}{l}32(33.0) \\
41(42.3)\end{array}$ & $153(640)$ \\
\hline & & $0(0.0)$ & $2(0.8)$ \\
\hline & $6(1$ & $9(9.3)$ & $16(6.7)$ \\
\hline
\end{tabular}

TABLE 4. Outcomes of 215 pediatric patients with hydrocephalus who underwent VPS placement preoperatively 
Clinical characteristics

\& Outcomes

Gender (M : F)

Ages (years)

Postop. VPS

Postop. ETV
Suprasellar (21)

$7.5 \pm 4.5$

$2(\overline{9} .5)$

$0(0.0)$
Location of tumors (n)

Pineal region (41) Fourth ventricle (153) $\quad \boldsymbol{p}$

\begin{tabular}{ccc}
$34: 7$ & $94: 59$ & $\boldsymbol{p}$ \\
$.2 \pm 4.2$ & $7.1 \pm 3.9$ & 0.021 \\
$0(0.0)$ & $1(0.7)$ & 0.010 \\
$0(0.0)$ & $1(0.7)$ & 0.042 \\
\hline
\end{tabular}

$.1 \pm 3.9$

$1(0.7)$

.010

-

\begin{tabular}{|c|c|c|c|c|}
\hline \multirow{2}{*}{$\begin{array}{l}\text { Clinical characteristics } \\
\& \text { Outcomes }\end{array}$} & \multicolumn{3}{|c|}{ Location of tumors (n) } & \multirow[b]{2}{*}{$p$} \\
\hline & $\begin{array}{c}\text { Suprasellar } \\
(1)\end{array}$ & $\begin{array}{c}\text { Pineal region } \\
(32)\end{array}$ & $\begin{array}{c}\text { Fourth ventricle } \\
\text { (7) }\end{array}$ & \\
\hline $\begin{array}{l}\text { Gender (M : F) } \\
\text { Ages (years) } \\
\text { Postop. VPS }\end{array}$ & $\begin{array}{c}1: 0 \\
15.0 \\
0(0.0)\end{array}$ & $\begin{array}{c}27: 5 \\
10.3 \pm 4.2 \\
3(9.4)\end{array}$ & $\begin{array}{c}3: 4 \\
7.0 \pm 3.4 \\
1(14.3)\end{array}$ & $\begin{array}{l}0.069 \\
0.078 \\
0.838\end{array}$ \\
\hline Postop. ETV & $0(0.0)$ & $0(0.0)$ & $0(0.0)$ & - \\
\hline
\end{tabular}

TABLE 6. Outcomes of 117 pediatric patients with hydrocephalus who did not undergo with VPS placement or ETV preoperatively

\begin{tabular}{|c|c|c|c|c|}
\hline \multirow{2}{*}{$\begin{array}{l}\text { Clinical characteristics } \\
\text { \& Outcomes }\end{array}$} & \multicolumn{3}{|c|}{ Location of tumors (n) } & \multirow[b]{2}{*}{$p$} \\
\hline & $\begin{array}{c}\text { Suprasellar } \\
(14)\end{array}$ & $\begin{array}{c}\text { Pineal region } \\
(24)\end{array}$ & $\begin{array}{c}\text { Fourth ventricle } \\
\text { (79) }\end{array}$ & \\
\hline $\begin{array}{l}\text { Gender }(M: F) \\
\text { Ages (years) } \\
\text { Postop. VPS } \\
\text { Postop. ETV }\end{array}$ & $\begin{array}{c}3: 11 \\
6.3 \pm 3.2 \\
4(28.6) \\
1(7.1)\end{array}$ & $\begin{array}{c}18: 6 \\
8.4 \pm 3.8 \\
6(25.0) \\
0(0.0)\end{array}$ & $\begin{array}{c}51: 28 \\
6.9 \pm 3.8 \\
14(17.7) \\
1(1.3)\end{array}$ & $\begin{array}{l}0.003 \\
0.155 \\
0.540\end{array}$ \\
\hline
\end{tabular}

\section{Discussion}

The mechanisms of hydrocephalus have not yet been fully illuminated, but it has been proven that the deterioration of CSF dynamics may play a role[2]. This study mainly discussed acquired hydrocephalus; some pathophysiological processes that affect ventricular outflow and subarachnoid absorption as well as brain compliance may contribute to the occurrence and development of this condition[10]. It has been reported in the literature that intraventricular hemorrhage is the most common cause of acquired hydrocephalus in infants, including premature babies. After this period, tumors become the most frequent etiology of acquired hydrocephalus, especially midline tumors in the fourth ventricle[2, 3, 10]. These tumors obstruct the CSF circulation, leading to acquired hydrocephalus, and therefore, this condition is classified as obstructive hydrocephalus. Similarly, we found that malignant midline intracranial tumors in pediatric patients usually lead to obstructive hydrocephalus, which is consistent with previous reports. Furthermore, by analyzing the patient characteristics, it was clear that the types of the tumor differed among locations, and the sex ratio and age at first surgery were also different for different types of tumors.

This large series demonstrated several findings regarding treatments as well. Direct tumor resection was able to re-establish CSF circulation and avoid a second operation. Nevertheless, this situation may be 
temporary, as hydrocephalus relapse can be caused by residual tumor tissue, blood clots, hemostatic material, or partial brain swelling, which are hard to predict. Therefore, if the tumors were directly resected after the diagnosis, without using any method to relieve the hydrocephalus in advance, there is a high probability that the hydrocephalus would immediately recur. This situation would not only interrupt subsequent radiotherapy or chemotherapy but also can be life-threatening in some cases. The treatment method cannot predict the subsequent changes in patient condition; hence, a specific method is not recommended in clinical practice. According to the outcomes of 117 patients who underwent direct tumor resection in this study, the recurrence rate of hydrocephalus was as high as $20.5 \%$, and the shortest duration was only 7 days. A retrospective study in 2018 found that the recurrence rate after direct resection of tumors in midline regions, such as posterior fossa tumors, in children was $8.4 \%$ [3], and this rate was even over $25 \%$ in other reports ${ }^{[21]}$.

The placement of a VPS is a simple and safe surgery, and this device can effectively relieve various kinds of hydrocephalus. According to reports, the perioperative mortality rate of VPS placement is under 1\%[22], and the estimated 30 -year VPS-related mortality rate is $5-10 \%[10,19]$; moreover, some reports showed that the overall survival rate after VPS placement was $70 \%$ at 1 year, $58 \%$ at 10 years, and $49 \%$ at 20 years[1], showing a decreasing trend year to year. According to our data analysis, the effectiveness of an initial VPS was $98.1 \%$ among 215 patients, and among those patients who failed initial ETV, a VPS can also solve the problem of hydrocephalus recurrence. Interestingly, a study in 2011 declared that patients with malignant tumors were less likely to experience VPS failure than those with benign tumors[20], which may mean that patients with malignant tumors should be more inclined to choose a VPS preoperatively. Nevertheless, the disadvantages of VPSs are obvious as well. Foreign materials can be implanted for a long time, but whether these materials can be removed eventually remains to be explored. Moreover, obstruction is the most common cause of mechanical shunt failure[1]. Previous studies have indicated that the rate of shunt infection is approximately $5-9 \%[11,12]$, and the first infection commonly occurs within 3 months after surgery.[14,27] With ETV, the problem of foreign material implantation is fundamentally avoided. The success rate of initial ETV ranges from $71 \%$ to over $90 \%[6,28]$, and the overall complication rate of ETV was shown to be $9 \%$ in recent meta-analyses[17, 25]. According to our data, the effectiveness of preoperative ETV was $90.0 \%$, which is consistent with previous reports. Regarding the disadvantages of this method, closure of the stoma secondary to gliosis usually presents within 2 years[4]; therefore, whether hydrocephalus can be continuously alleviated in the postoperative and subsequent treatment period is unknown, necessitating the need for larger survey samples and a longer follow-up.

As a consequence, when choosing between VPS and ETV, we should not place more emphasis on ETV or even disregard VPS. According to our data analysis, VPS and ETV were equally able to relieve hydrocephalus, and the effectiveness of both was over $90 \%$; however, upon deeper analysis, we found that preoperative VPS placement followed by resection and postoperative VPS placement was significantly different from preoperative ETV followed by resection and postoperative VPS placement, revealing that preoperative VPS placement led to a lower hydrocephalus recurrence rate and was more 
effective. One of the longest follow-up surveys of pediatric patients with hydrocephalus declared that the long-term survival rate with a VPS was higher than that of ETV[1]; moreover, ETV failure occurred sooner than VPS failure according to another former study[5]. These previous results support our conclusion. Regarding those who are inclined to replace VPS placement with ETV, such views are mostly based on the skepticism and controversy about the higher complication rate, but even if shunt malfunctions and infections occur, careful aseptic techniques can decrease the rate of VPS-related infection[13], and the coating material and shunt design are also undergoing updates to reduce the possibility of obstruction[10]. Both VPS placement and ETV have their own advantages and disadvantages; therefore, it is highly unlikely that VPS placement will become an obsolete therapy in the foreseeable future; on the contrary, VPSs are crucial.

\section{Conclusion}

Malignant midline intracranial tumors in pediatric patients usually lead to obstructive hydrocephalus, and reliable and stable relief of hydrocephalus during the whole treatment period is the goal of comprehensive treatment for children.

We rarely recommend direct resection on account of a high recurrence rate. VPSs and ETV are two kinds of safe and effective treatments for obstructive hydrocephalus caused by midline tumors malignant, and both approaches can lay a solid foundation to ensure the safety of follow-up chemoradiotherapy.

Therefore, VPSs and ETV can achieve a similarly high rate of effectiveness in the treatment of obstructive hydrocephalus secondary to malignant midline intracranial tumors during the perioperative period. Nevertheless, many patients who undergo initial ETV may experience hydrocephalus relapse and need to be rescued by a VPS.

\section{Declarations}

Funding: This work was supported by the National Natural Science Foundation of China (81870834 and 81502150), the National Key Research and Development Project (2017YFE0121200) , Special Fund for Pediatrics from Beijing Hospitals Authority (XTYB201817), the Capital Health Development Research Project (2020-2-2045), Beijing Advanced Innovation Center for Big Data-Based Precision Medicine, Beihang University, Beijing, China National Natural Science Foundation of China (81930048), Capital Characteristic Clinical Application Project (Z181100001718196), and Beijing National Key Technology Research and Development Program of the Ministry of Science and Technology of China (2014BAI04B01, 2015BAI12B04 and 2013BAI09B03).

Conflict of interest: The authors report no conflicts of interest concerning the materials or methods used in this study or the findings specified in this paper.

Availability of data and material: All data generated or analysed during this study are included in this article. 
Code availability: Not applicable.

Ethics approval: Not applicable.

Consent to participate: Not applicable.

Consent for publication: Not applicable.

Authors' contributions: Yunwei Ou and Kaiyu Fan contributed equally to this work. Jian Gong found out the problem according to clinical routine work. Zhiming Liu, Zhiyi Liao, Heng Zhang, Zhicen Li, Wenjian Zheng and Xueyi Guan collected the patients' data. Yunwei Ou analyzed and interpreted the data, Kaiyu Fan was a major contributor in writing the manuscript. All the authors read and approved the final manuscript.

\section{References}

1. Beuriat PA et al (2017) Hydrocephalus treatment in children: long-term outcome in 975 consecutive patients. J Neurosurg Pediatr 20(1):10-18

2. Chan DYC et al., Emergency endoscopic third ventriculostomy for blocked shunts? Univariate and multivariate analysis of independent predictors for failure. J Neurosurg, 2018: p. 1-7

3. Chel'diev BZ, Kushel YV, Demin MO, [Implantation of VPS in the early postoperative period following removal of posterior cranial fossa tumors in children]. Zh Vopr Neirokhir Im N N Burdenko, 2018. 82(4): pp 81-86

4. Deopujari CE, Karmarkar VS, Shaikh ST (2017) Endoscopic Third Ventriculostomy: Success and Failure. J Korean Neurosurg Soc 60(3):306-314

5. Dewan MC et al (2017) The durability of endoscopic third ventriculostomy and ventriculoperitoneal shunts in children with hydrocephalus following posterior fossa tumor resection: a systematic review and time-to-failure analysis. J Neurosurg Pediatr 19(5):578-584

6. Duru S et al (2018) Successful endoscopic third ventriculostomy in children depends on age and etiology of hydrocephalus: outcome analysis in 51 pediatric patients. Childs Nerv Syst 34(8):15211528

7. Grand W et al (2016) Endoscopic Third Ventriculostomy in 250 Adults With Hydrocephalus: Patient Selection, Outcomes, and Complications. Neurosurgery 78(1):109-119

8. Jiang L, Gao G, Zhou Y (2018) Endoscopic third ventriculostomy and ventriculoperitoneal shunt for patients with noncommunicating hydrocephalus: A PRISMA-compliant meta-analysis. Med (Baltim) 97(42):e12139

9. Jones RF, Stening WA, Brydon M (1990) Endoscopic third ventriculostomy Neurosurgery 26(1):8691; discussion $91-2$

10. Kahle KT et al (2016) Hydrocephalus in children. The Lancet 387(10020):788-799 
11. Kandasamy J et al (2011) Antibiotic-impregnated ventriculoperitoneal shunts-a multi-centre British paediatric neurosurgery group (BPNG) study using historical controls. Childs Nerv Syst 27(4):575581

12. Kestle JR et al (2011) A standardized protocol to reduce cerebrospinal fluid shunt infection: the Hydrocephalus Clinical Research Network Quality Improvement Initiative. J Neurosurg Pediatr $8(1): 22-29$

13. Kim HS et al (2019) Clinical outcome of cerebrospinal fluid shunts in patients with leptomeningeal carcinomatosis. World J Surg Oncol 17(1):59

14. Kulkarni AV, Drake JM, Lamberti-Pasculli M (2001) Cerebrospinal fluid shunt infection: a prospective study of risk factors. J Neurosurg 94(2):195-201

15. Maller VV, Gray RI, Noncommunicating Hydrocephalus. Seminars in Ultrasound, CT and MRI (2016) 37(2): p. 109-119

16. Miwa T et al (2015) Neuroendoscopic biopsy and the treatment of tumor-associated hydrocephalus of the ventricular and paraventricular region in pediatric patients: a nationwide study in Japan. Neurosurg Rev 38(4):693-704

17. Morelli D et al (2005) Persistent hydrocephalus after early surgical management of posterior fossa tumors in children: is routine preoperative endoscopic third ventriculostomy justified? J Neurosurg 103(3 Suppl):247-252

18. Prusseit $\mathrm{J}$ et al (2009) Epidemiology, prevention and management of ventriculoperitoneal shunt infections in children. Pediatr Neurosurg 45(5):325-336

19. Reddy GK et al., Ventriculoperitoneal shunt surgery outcome in adult transition patients with pediatric-onset hydrocephalus. Neurosurgery, 2012. 70(2): p. 380-8; discussion 388-9

20. Reddy GK et al (2011) Ventriculoperitoneal shunt complications in hydrocephalus patients with intracranial tumors: an analysis of relevant risk factors. J Neurooncol 103(2):333-342

21. Sainte-Rose C et al (2001) Management of hydrocephalus in pediatric patients with posterior fossa tumors: the role of endoscopic third ventriculostomy. J Neurosurg 95(5):791-797

22. Smith ER, Butler WE, Barker FG 2 (2004) -hospital mortality rates after ventriculoperitoneal shunt procedures in the United States, 1998 to 2000: relation to hospital and surgeon volume of care. J Neurosurg 100(2 Suppl Pediatrics):90-97 In, (

23. Stein SC, Guo W (2008) Have we made progress in preventing shunt failure? A critical analysis. J Neurosurg Pediatr 1(1):40-47

24. Symss NP, Oi S (2015) Is there an ideal shunt? A panoramic view of 110 years in CSF diversions and shunt systems used for the treatment of hydrocephalus: from historical events to current trends. Childs Nerv Syst 31(2):191-202

25. Tamburrini G et al (2008) Endoscopic third ventriculostomy: the best option in the treatment of persistent hydrocephalus after posterior cranial fossa tumour removal? Childs Nerv Syst 24(12):1405-1412 
26. Texakalidis $P$ et al (2019) Endoscopic third ventriculostomy versus shunt for pediatric hydrocephalus: a systematic literature review and meta-analysis. Childs Nerv Syst 35(8):1283-1293

27. Walters BC (1992) Cerebrospinal fluid shunt infection. Neurosurg Clin N Am 3(2):387-401

28. Won SY et al (2020) Management of hydrocephalus after resection of posterior fossa lesions in pediatric and adult patients-predictors for development of hydrocephalus. Neurosurg Rev 43(4):1143-1150

\section{Figures}



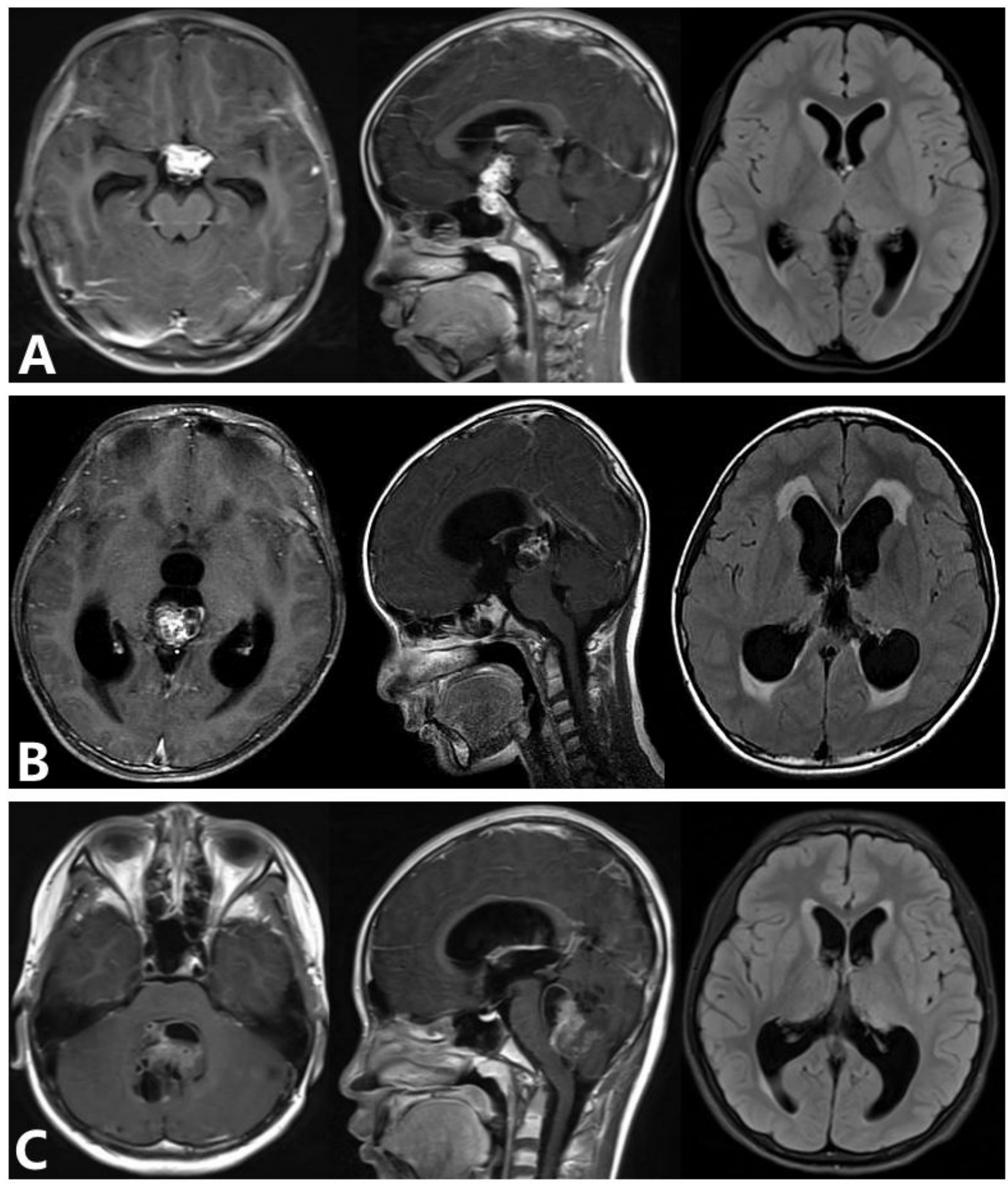

Figure 1

Preoperative magnetic resonance imaging (MRI) of the included children. There're axial, sagittal enhanced images and fluid attenuated inversion recovery (FLAIR) from left to right, respectively. A: Tumor in the suprasellar, its pathology was mixed germ cell tumor; B: Tumor in the pineal region, and the pathology was germinoma; C: Tumor in the fourth ventricle, and its pathology was medulloblastoma. All the midline tumors caused obstructive hydrocephalus with paraventricular edema. 